

TITRE: LA PERCEZIONE DELLA NORMA LINGUISTICA ATTRAVERSO IL PERIODICO LA CRUSCA PER VOI Auteur(s): DALILA TASSONE, UnIVERSITÀ DEGLI STUDI DI MESSINA

ReVue: CirCula, NumÉro 1, PAGES 130-152

ISSN: 2369-6761

DiRecteurs: Wim Remysen et SABINE SchWARZE

URI: HTTP://HDL.HANDLE.NET/11143/8003

DOI: $10.17118 / 11143 / 8003$ 


\title{
La percezione della norma linguistica attraverso il periodico La Crusca per voi
}

Dalila Tassone, Università degli studi di Messina dalilatassone @ alice.it

\begin{abstract}
Riassunto: Dedicato «alle scuole e agli amatori della lingua», ai lettori interessati e preoccupati delle sorti della lingua nazionale, il periodico semestrale La Crusca per voi esce per la prima volta nell'ottobre 1990 con una Giustificazione e Un po' di storia, in cui il fondatore Giovanni Nencioni spiega le ragioni dell'istituzione di un vero e proprio «consultorio linguistico», destinato ad accogliere le richieste, i dubbi, le perplessità sul corretto impiego della lingua italiana proveniente da persone di ogni livello sociale e culturale. Scopo di questo lavoro è studiare la tipologia dei quesiti posti agli accademici della Crusca, con particolare attenzione a fenomeni di carattere lessicale, negli ultimi diciotto numeri del periodico (dal 2005 al 2013, con qualche prelievo da uscite precedenti). Conclude il lavoro una breve indagine a campione sulla diffusione e la traducibilità di alcuni termini stranieri in italiano.
\end{abstract}

Parole chiave: norma linguistica; italiano standard; lessico; termini stranieri

Abstract: Ever since its first publication in October 1990, La Crusca per Voi is dedicated to language amateurs and readers interested in the destiny of the Italian national language. As pointed out by its founder, Giovanni Nencioni, La Crusca per Voi functions as a «language counselor» and gathers information on doubts and misgivings about the correct use of the Italian language. The aim of this study is to analyze different categories of lexical issues, such as synonyms, neologisms and foreign words, that are raised by the readers of La Crusca per Voi. The study ends with a short sample survey on the prevalence and translatability of some foreign words in Italian.

Keywords: linguistic norm; standard Italian; lexicon; foreign words 


\section{Introduzione}

Il guaio l'hanno fatto, temporibus illis, un purismo intransigente e una grammatica logicizzante, che hanno ingenerato la convinzione che la norma sia un logos astratto, metafisico, calato in un catechismo grammaticale, mentre la norma è dentro i testi degli scrittori e i discorsi dei parlanti e spesso si offre loro come un fascio di possibilità alterne, di scelte, ed essi possono più o meno consapevolmente, nel corso del tempo e nel mutare di certe condizioni, confermarla o modificarla. (Nencioni, 1989: 227)

Così si esprimeva, nel 1989, Giovanni Nencioni nel saggio dal titolo emblematico Disperare dell'italiano? e da qui si è deciso di prendere le mosse per affrontare la delicata questione delle norma linguistica, ampiamente dibattuta dagli studiosi, ma che interessa sempre più i parlanti, i veri e propri fruitori della lingua.

Per farlo, ci serviremo del periodico La Crusca per voi (d'ora in avanti, CPV), foglio dell'Accademia della Crusca, che dal 1990 si pone «a diretto contatto con gl'indiretti destinatari del suo operare - l'intero popolo italiano - tramite l'istituzione di un consultorio linguistico «come servizio nazionale» (Nencioni, CPV. 1. Ottobre 1990. Giustificazione)․․

\section{Sul concetto di norma linguistica}

Prima di entrare in medias res, vale la pena procedere con un sintetico excursus sul concetto di norma.

Dalla strutturazione tripartita sistema, norma, parole di Eugenio Coseriu (1971) ricaviamo una definizione di norma che privilegia l'aspetto sociale della lingua, una norma che «può considerarsi come sistema di realizzazioni obbligatorie, di "imposizioni" sociali e culturali, e dipende dall'estensione e dalla indole della comunità considerata» (Coseriu, 1969: 250). Non si tratta

della norma nel senso corrente, stabilita od imposta secondo criteri di correttezza o di valutazione soggettiva di quel che viene espresso, bensì della norma obiettivamente constatabile in una lingua, la norma che seguiamo necessariamente se vogliamo essere membri di una comunità linguistica. (Coseriu, 1971: 76)

Secondo lo studioso tedesco, ancora, la norma deve «constatare come si dice» senza indicare «come si deve dire», opporre il «normale» all'«anormale» e non il «corretto» da ciò che è «scorretto» (Coseriu, 1971: 76).

Dalle astrazioni coseriuane di sistema e norma, Serianni muove per introdurre «il giudizio linguistico dei parlanti» (Serianni, 2006: 37), la reazione dell'utente di una lingua dinanzi alla violazione di

1. Le cifre a seguito dell'abbreviazione indicano il numero del periodico, il numero progressivo del quesito, il mese e l'anno di pubblicazione del foglio. 
norme comunemente condivise, particolarmente di quella «norma linguistica interiorizzata» stratificatasi «non tanto sulla base della propria esperienza di parlante, quanto sull'immagine di lingua che si è formata negli anni di scuola» (Serianni, 2006: 52).

Dal punto di vista prescrittivo, nell'oscillazione dicotomica «tra i due poli "giusto"/“sbagliato"», lo studioso individua una «zona grigia», in cui il parlante può avere dubbi o incertezze sul corretto impiego della lingua (Serianni, 2006: 42), perché:

Le controversie non nascono quando la norma è stabile e indiscussa, ma quando essa è in corso di trasformazione o quando c'è coesistenza di più norme parallele, adatte a diverse situazioni comunicative. [...] È l'uso attuale a dar fondamento alla norma linguistica. (Serianni: CPV, 2. Aprile 1991)

Di qualche anno di tardi, le considerazioni di Massimo Palermo, a conclusione della trattazione sulla norma linguistica attraverso i manuali redazionali, gli strumenti di consultazione editi dalle case editrici, dalle stampe periodiche, dalle sedi di enti pubblici:

[...] negli ultimi decenni abbiamo assistito ad una progressiva frantumazione della norma. Nella coscienza dei parlanti, all'accettazione di una norma univoca, [posseduta da un numero limitato di persone e adatta ai piani alti della comunicazione], si è sostituita la consapevolezza della legittima coesistenza, anche nello scritto, di più norme, ciascuna adeguata a una particolare situazione comunicativa. (Palermo, 1995: 113)

Per Marazzini il «delicato concetto di norma» è quello che

abbiamo visto nascere come riconoscimento dell'armonia presente nei testi e poi abbiamo visto sfumare via via, trasformandosi nella libertà individuale e, nelle ultime fasi storiche, perdersi nel concetto di varietà. (Marazzini, 2006: 95)

Giuseppe Antonelli, in un viaggio nell'«italiano della società di comunicazione» (questo il titolo del suo contributo), scrive:

Perché in una lingua viva e parlata da (quasi) tutti gli italiani in (quasi) tutti gli usi comunicativi, la norma si rifrange in una pluralità di norme. E se proprio dobbiamo avere presente un modello, non guarderemo ad una norma derivata dalla sommatoria di tanti ipse dixit, ma a una norma intesa come normalità, come varietà neutra, non marcata in senso stilistico o geografico. (Antonelli, 2007: 46) 
«La norma non è una immutabile tavola dei comandamenti», afferma Renzi (2012: 172), ma ha una stretta rispondenza con i parlanti, «che [...] possono portare avanti le innovazioni fino a farle diventare norma, oppure abbandonarle a un certo punto, frenare i cambiamenti fino a provocare la vittoria delle forme della norma» (Renzi, 2012: 170).

Da ultimo, Serianni (2014) torna sul concetto di errore, facendo riferimento alle considerazioni di Sgroi (2010). In chiusa alla panoramica sulle definizioni di uso, norma (regola), errore in tredici dizionari dal 1956 al 2008, Sgroi afferma:

Il problema dei (neo)puristi è quello di etichettare come «errori» usi linguistici che al massimo sono etichettabili come «sbagli», sia perché semanticamente del tutto comprensibili sia perché a un tempo prodotti da parlanti per definizioni non-subalterni, colti o mediamente colti, e quindi non attribuibili all'italiano cosiddetto «popolare». (Sgroi, 2010: 26)

Serianni richiama nuovamente alla necessità di considerare «oltre alle indicazioni di classiche fonti della norma linguistica come dizionari, grammatiche e, soprattutto, tradizione scolastica, la reattività dei parlanti», ove questi ultimi sentano violato il «comune sentimento della lingua» (Serianni, 2004: 102).

\section{Il Periodico La Crusca per voi}

Alla consapevolezza dello studioso della variabilità della norma linguistica e dell'impossibilità, dunque, di fornire regole sistematiche e incontrovertibili, si contrappone la necessità da parte degli utenti di una prescrizione di forme, di una serie di classificazioni che regolino l'uso lasciando poco spazio all'arbitrio.

A questo risponde un'iniziativa dell'Accademia della Crusca destinata, come recita il frontespizio, «alle scuole e agli amatori della lingua»: si tratta del periodico La Crusca per voi, nato, preliminarmente, come una sorta di risposta alla generosità di tutte quelle istituzioni, associazioni pubbliche e private, istituti bancari, industriali, professionali, ma anche singoli cittadini che, col proprio contributo, hanno risollevato le sorti economiche dell'Accademia della Crusca colpita, negli anni 1988 e 1989, da una grossa crisi finanziaria che ha drasticamente ridotto l'attività di ricerca.

Il periodico è diventato, nel corso degli anni e delle uscite (del maggio 2014 è, ad oggi, l'ultima pubblicazione, la numero 48), un vero e proprio punto di riferimento per quegli «amatori» della lingua, che sulla lingua si interrogano e sulla quale sperano di ricevere il responso autorevole degli «espertissimi» (CPV. 1. Ottobre 1990), impegnanti in una «conversante consulenza» (Accademia della Crusca, 1995: 5. Presentazione di Nencioni) coi lettori.

Per questa rassegna sulle curiosità, le incertezze che giungono ormai da ventiquattro anni alla redazione dell'Accademia della Crusca, sulla scorta delle raccolte già pubblicate (ovvero La Crusca 
risponde del 1995 e la recente La Crusca risponde. Dalla carta al web del 2013), cercheremo di isolare le tematiche più frequenti in fatto di dubbi linguistici negli ultimi nove anni, dal 2005 all'ottobre 2013 (per un totale di 18 numeri), con qualche incursione in numeri antecedenti, soprattutto per le questioni, assai dibattute, dei neologismi e dei forestierismi.

Sulla scia di Serianni, ci interesseremo «[al]la tipologia dei quesiti e [al]]'immagine della lingua che li ispira» (Serianni, 2006: 48) più che alle risposte degli studiosi, seppur di volta in volta faremo nostre le parole dei consulenti. Le lettere, dunque, rielaborate dalla redazione e «doverosamente potate di riferimenti al personale vissuto dei mittenti» (Serianni, 2006: 47), costituiranno il corpus di questa prima parte del lavoro.

La trattazione procederà per sottoparagrafi; distingueremo tra fenomeni sinonimici, di risemantizzazione, neologismi - o presunti tali, forestierismi.

\subsection{Sinonimia}

Convinti che la norma riguardi anche il lessico, «con tutte le sue, talora sottili, deflessioni dall'uso» (Serianni, 2008: 61), segnaliamo una serie di quesiti nel campo della sinonimia.

«Perché ci sono due forme di participio passato per il verbo "dissolvere" e che differenza di significato c'è tra "dissoluto" e "dissolto" nell'italiano contemporaneo e nella lingua antica?», chiede un signore di Lecce (CPV, 44.4. Aprile 2012); una lettrice vuol avere ragguagli sulla differenza di significato degli aggettivi previdenziale e provvidenziale e sulla loro corretta distribuzione nell'uso (CPV, 40.5. Aprile 2010), e, ancora, una domanda riguarda l'ambito d'uso dei termini prelevamento e prelievo (CPV, 43.9. Ottobre 2011).

Talvolta il lettore, accanto al quesito diretto all'accademico, propone una propria interpretazione al problema, come nel caso che segue:

«Collaborare» e «cooperare» sono sinonimi? Sembra che il primo comporti che tutti si attivino per un compito («collaborare»), il secondo una suddivisione di incarichi in vista del risultato finale («cooperare»). (CPV, 44.3. Aprile 2012)

Qualche curiosità investe i linguaggi specifici: un'appassionata di lingua del diritto chiede la differenza di significato tra inerzia e inadempimento (CPV, 31.5. Ottobre 2005); una ragazza di Bolzano vuol sapere se manuale e prontuario siano termini coincidenti (CPV, 43.4. Ottobre 2011) e, nello stesso numero 43 del foglio, si chiede se prelevamento e prelievo vadano utilizzati in contesti d'uso diversi (CPV, 43.9. Ottobre 2011). 
Un ultimo quesito riguarda il grado di sinonimia in italiano dei verbi accadere, succedere, capitare. Il richiedente francese aggiunge: «I sinonimi non sono mai esattamente sinonimi, vorrei sapere se esistono differenze d'uso per questi verbi e se ci sono vincoli particolari nella scelta dell'uno e dell'altro» (CPV, 38.9. Aprile 2009). «"Capitare, succedere e accadere”, argomenta Giada Mattarucco, «sono "fratelli" abbastanza somiglianti», ma, aggiungiamo noi, non «gemelli», per dirla con Pontiggia che la stessa Mattarucco cita nella sua risposta².

Qualche considerazione. La variazione semantica è strettamente connessa a quella sociolinguistica. Nei casi di specie assistiamo alla pressione esterna di fattori condizionanti: una certa specializzazione linguistica che porta a interrogarsi su inerzia e inadempimento (in ambito giuridico), piuttosto che su manuale e un prontuario (in ambito didattico); un incremento della cultura del parlante che vuol essere certo di impiegare i termini nel corretto contesto d'uso (quella variabile diafasica che richiama ad un impiego pertinente delle voci, ma anche diastatica, come si suggerisce in Rossi e Ruggiano 2013) $)^{3}$ per cui non si accontenta di usare indifferentemente prelevamento o prelievo se impegnato in una operazione bancaria o di parlare, senza distinzione, al proprio gruppo di lavoro di cooperazione e collaborazione.

Ancora, incide, come vedremo nel caso che segue, la penetrazione di termini stranieri, che modificano la semantica di alcune voci italiane:

Desidererei avere chiarimenti a proposito del significato che assumono i termini oramai comuni in campo giornalistico (e non solo) di «intrigante» (nel senso anglosassone di chi suscita fascinosa curiosità) e «suggestione» (nel senso anglosassone di suggerimento). (CPV, 32.2. Aprile 2006)

\subsection{Risemantizzazione}

Una signora nota la recente diffusione mediatica della parola tesoretto, usata per indicare l'extra-gettito delle finanze statali, e chiede se si tratti di un neologismo a tutti gli effetti o un nuovo significato assunto da una parola esistente (CPV, 35.5. Ottobre 2007). Un lettore pescarese segnala usi a suo avviso impropri del verbo paventare: gli sembra, infatti, che il termine sia utilizzato come sinonimo di avere intenzione, avere in animo di nella frase «La Regione paventa la realizzazione di un nuovo ospedale»; «Si tratta di un nuovo significato assunto dal termine?», conclude (CPV, 42.2. Aprile 2011).

2. «Non ho mai creduto alla esistenza dei sinonimi, né alla uguaglianza dei gemelli. Ė apparente, anche nei monozigoti. [...] se scrivendo chiedete a una persona [...] quale tra due parole preferisce e lei risponde "Ė lo stesso", cancellatela per l'eternità dall'albo dei lettori, nonché, a maggior ragione, degli scrittori» (Pontiggia, 2009: 25)

3. «Non bisogna frettolosamente attribuire le ragioni della distinzione tra quasi-sinonimi all'asse diafasico: anche quando uno scarto formale è avvertito, esso poggia su basi semantiche, che si rispecchiano prima di tutto sull'asse diastratico» (Rossi e Ruggiano, 2013: 285). 
Alla segnalazione della prima lettrice circa la diffusione della parola tesoretto, Valeria Della Valle risponde che la voce, già attestata nel GDLI con il significato di «camera blindata di una banca, cassaforte, caveau», entra nell'uso a dal 2007, sotto la spinta forte dei giornalisti che hanno preferito usare il diminutivo tesoretto dalla connotazione «più accattivante quasi familiare», rispetto al burocratico extra-gettito.

Per quel che riguarda la seconda richiesta, sarà interessante notare l'assoluta mancanza di contestualizzazione della frase da parte del lettore, che rende impossibile una risposta univoca al quesito, ma che consente di tornare all'aspetto che maggiormente ci interessa: la sollecitazione del parlante poggia sull'individuazione di una singola parola (paventare) percepita come deviazione dalla norma, che, in questo caso, si identifica con significato comunemente attribuito al verbo, ovvero aver paura, temere. Anche in questo è possibile individuare quell'«atteggiamento iper-razionalistico» di cui dice Serianni, fondato su un'immagine astratta della norma, «sottratta alla variabilità degli usi concreti» (Serianni, 2006: 48), che, nel caso di specie, si palesa in una decontestualizzazione dell'oggetto della richiesta stessa ${ }^{4}$.

\subsection{Neologismi}

Se un calco semantico è talvolta percepito come un neologismo (come il tesoretto di cui sopra), il dubbio che un termine sia una neoformazione si insinua nel parlante non appena lo stesso non trovi riscontro sui vocabolari.

"La parola "megaevento"», chiede una lettrice, «è un neologismo? E, se così è, perché non è inserita in nessun dizionario?» (CPV, 33.2. Ottobre 2006)5; una signora siciliana, presumibilmente un avvocato, chiede se esista davvero il verbo innucleare, che ha incontrato in una sentenza del 2005 e che non trova registrato in nessun dizionario (CPV, 38.4. Aprile 2009).

Interessante, per quest'ultimo caso riportato, la risposta di Sabatini: dopo aver fatto ammenda per il fatto che la parola non è registrata nel vocabolario da egli stesso curato, lo studioso «per avere maggiore certezza sull'esistenza e sul significato di una parola» si appella, non senza un «ahimè» assai eloquente, «a quel gran dizionario dei dizionari (si fa per dire) che è la rete globale» (CPV, 38.4. Aprile 2009)6.

4. «Purtroppo la frase è stata estrapolata dal contesto in cui era e, così com'è, non appare necessariamente scorretta», scrive Raffaella Setti in risposta.

5. Vittorio Coletti e Manuela Manfredini, in risposta ad una serie di «neologismi o supposti tali», ritengono che formazioni con pluri- e mega-siano neologismi «che non è neppure necessario registrare in un dizionario, perché il loro significato è pienamente desumibile da quello dei loro costituenti» (CPV, 33.2. Ottobre 2006).

6. Il verbo, insieme a innuclea, innucleato, ha il suo primo esempio nel catalogo dell'VIII biennale d'arte di Venezia del 1909 e risulta ampiamente attestato in testi di materi giuridici, scientifici, di saggistica. 
Il verbo promozionare «esiste o non esiste?» (CPV, 41.9. Ottobre 2010); una lettrice, dopo aver sentito il termine in bocca ad un ministro, lamenta il fatto di non averlo trovato sul vocabolario, come se, assumendo le parole del risponditore Salvatore Claudio Sgroi, «un vocabolario dovesse o potesse accogliere tutte le parole e tutti i significati adoperati da una comunità (massa) di parlanti di circa 60 milioni di italiani» (CPV, 41.9. Ottobre 2010).

Un lettore straniero chiede chiarimenti sull'esistenza e sul significato del verbo rammostrare, usato normalmente da avvocati e magistrati, ma che non si trova registrato nei maggiori vocabolari della lingua italiana. «Si tratta di una parola inventata di sana pianta da qualcuno e poi usata da altri come un gregge di pecorelle?» (CVP, 31. 12. Ottobre 2005), tuona il lettore attribuendo ai parlanti scarsa capacità di discernimento quanto ad usi lessicali .

Questo genere di quesiti mostrano quanto sia radicata nei parlanti l'immagine di lingua formatasi negli anni di scuola, quella che Serianni (2007) ha anche definito «norma "sommersa"», e che, soprattutto per scriventi adulti, ha la propria auctoritas nelle grammatiche e, ancor di più, nei dizionari. Per verificare l'esistenza di una parola ci si appella al vocabolario, «consigliere fidato» (Serianni, 2006: 52), rispetto al quale è più facile dubitare dell'esistenza di un termine che pensare ad una lacuna dei curatori o, come sarebbe più auspicabile, ad un movimento naturale delle lingue che naturalmente porta all'incremento lessicale.

Già nel foglio 11 del 1995 un lettore aveva chiesto se fosse lecito usare parole italiane «non attestate in scritti o in vocabolari» (CPV, 11.5. Ottobre 1995). La norma degli scrittori, dunque, da una parte - ma Vittorio Coletti ci aveva ricordato che «la letteratura ha cessato di condizionare e orientare la lingua» (Coletti, 1989: 11) ${ }^{8}$ - e quella dei vocabolari dall'altra, che, afferma però Nencioni, «sono i notai, non i creatori della lingua» (CPV, 11.5. Ottobre 1995).

Per chiudere la questione sui neologismi, occorre evidenziare ancora qualche caso, in cui il lettore si improvvisa «onomaturgo» (Migliorini, 1975).

Gli allievi di un liceo scientifico pugliese avevano proposto tecnocida per «colmare la lacuna, nell'italiano, della parola che indichi, accanto a "matricida", "patricida", "fratricida", "infanticida", "uxoricida", l'uccisore dei figli» (CPV, 18.5. Aprile 1999)9'; a questa, si aggiunge la lunga e puntuale richiesta di un

7. Per Bice Mortara Garavelli, la voce non è un tecnicismo giuridico, ma può ascriversi a quelli che Serianni definisce «tecnicismi collaterali», ovvero, «espressioni stereotipiche non necessarie, a rigore, alle esigenze, della denotatività scientifica, ma preferite per la loro comunicazione tecnica» (CPV, 31.12. Ottobre 2005).

8. La lingua, continua Coletti, «cerca altrove le proprie autorità: mass media, pubblicità, industria, spot, politica, ecc. tengono oggi lo scettro linguistico che in passato spettava a poeti e romanzieri» (Coletti, 1989: 11).

9. La proposta non soddisfa pienamente Nencioni sia perché «in una serie di parole schiettamente latine e proprie della famiglia romana introduce una parola composta di un prefissoide greco "tecno-", designante i figli, seguito dal " -cida", schiettamente latino», sia perché i nostri dizionari sono ricchi di composti in cui prefissoide «tecno-» ha il significato di «arte», «tecnica» e «quindi si crea con l'altro formalmente identico un rischio di omonimia che nel campo delle lingue scientifiche dobbiamo evitare» (CPV, 18.5. Aprile 1999). 
laureato cosentino che chiede se il titolo Cronotopia dato alla propria tesi di laurea - titolo chiarito dal sottotitolo Soggetti, mutamenti, politiche del tempo. Calendari di donne e uomini - possa essere un neologismo conveniente (CPV, 7.6. Ottobre 1993) ${ }^{10}$. Una lettrice romana vorrebbe proporre il neologismo video-ascolto o video udienza in sostituzione della parola anglo latina audience (CPV, 9.13. Aprile 1994) ${ }^{11}$ e, ancora, richiedente casertano sostituirebbe volentieri neolemma a neologismo (CPV, 18.5. Aprile 1999) $)^{12}$.

Tornando ad esempi più recenti, troviamo la richiesta di un lettore che vuol sapere se rosicare e rosicone siano neologismi (CPV, 33.2. Ottobre 2006); l'ultimo quesito sull'argomento riguarda un fenomeno di costume proprio della televisione italiana: «Ho sentito in televisione il neologismo "tronista", di cui però non trovo tracce nei dizionari. Cosa significa e da dove ha origine la parola?» (CPV, 35.3. Ottobre 2007).

Quanto alla prima richiesta, la voce, attestata sin dal XVII secolo col significato di «rodere, intaccare qualcosa», ha assunto il significato di «rodersi dall'invidia, dalla rabbia» in ambito romanesco come dimostrano le occorrenze in un sonetto di Gioacchino Belli (il numero 1593: «Pija su e rosica») e nel capolavoro di Gadda, Quer pasticciaccio brutto de via Merulana («un'invidia che je rosicava er fegato»). Si tratterebbe, dunque, di una risemantizzazione di una voce esistente attraverso una parlata regionale che è uscita dall'area d'uso centro-italiana in tempi relativamente recenti, quando il calciatore Gennaro Gattuso ha dato dei «rosiconi» ai giornalisti francesi all'indomani della sconfitta della Francia ai mondiali del 2006.

Il sostantivo tronista oggetto del secondo quesito si è diffuso con il significato di «partecipante ad uno spettacolo televisivo che si presta a essere messo al centro dell'attenzione, su un trono appunto, divenendo oggetto di corteggiamento». Legittimamente, avverte Valeria Della Valle nella risposta, la voce non ha ancora trovato ospitalità nei principali vocabolari italiani perché «i redattori dei repertori lessicografici aspettano prudentemente che il termine entri nell'uso corrente, prima di accoglierlo nei loro lemmari» (CPV, 35.3. Ottobre 2007). Era il 2007; appena due anni dopo il termine, apparso per la prima volta in un articolo di Sebastiano Messina sulla Repubblica del 27 gennaio 2004, si è affacciato nel panorama lessicografico italiano, accolto nello Zingarelli 2009.

10. La risposta è affidata a Giovanni Nencioni che sottolinea la difficoltà e la rarità nella creazione di parole totalmente nuove. Per il caso di specie, lo studioso ricorda che il termine esiste già nella fisica della relatività «ad indicare uno spazio che include la dimensione del tempo» e che il tecnicismo «è passato nella semiotica ad indicare, ad es., lo spazio-tempo del narratore e del narrato» (CPV, 7.6. Ottobre 1993).

11. Ancora Nencioni si rivela favorevole ad impiegare un termine nella nostra lingua se perfettamente sinonimico al termine inglese; promuove, dunque, il composto videoudienza perché «anche se formato con un prefissoide d'importazione inglese, ma di origine latina, appare italiano e non offre possibilità di equivoco» (CPV, 9.13. Aprile 1994).

12. «Neolemma», informa Nencioni nella risposta, «dovrebbe così significare "nuova parola di dizionario collocata in esponente" e niente avrebbe a che vedere con neologismo il cui significato è assai più ampio e connesso con l'insieme della lingua, che accoglie neoformazioni quando si producono concetti od oggetti nuovi che occorre denominare» (CPV, 18.9. Aprile 2009). 


\subsection{Forestierismi}

Se per quanto riguarda i neologismi, i lettori sembrano mossi da una sana curiosità verso il nuovo e la vis polemica si placa laddove qualcuno si improvvisa addirittura onomaturgo, per dirla ancora con Migliorini, una vera a propria preoccupazione sembra muovere la penna di chi si interroga e interroga sui forestierismi.

Sedici richieste in diciotto numeri rilevano un allarmismo dei parlanti rispetto alla penetrazione di forestierismi in italiano, che poco si distanzia dal morbus anglicus che il medico Castellani aveva diagnosticato alla nostra lingua già dal $1987^{13}$.

I quesiti in cui più evidente è la preoccupazione sono quelli che con maggiore genericità pongono il problema dei forestierismi: nella prima uscita del 1990 un gruppo di alunni di una scuola media di Mantova si interroga sulla liceità dell'utilizzo nel linguaggio scritto di termini stranieri invece dei corrispondenti italiani (CPV, 1.7-9. Ottobre 1990); una signora della provincia di Bergamo scrive: «Come mai i vocaboli stranieri, che oggi imbastardiscono la nostra lingua, vengono spesso usati con un significato che non corrisponde nemmeno per approssimazione a quello originale?» (CPV, 4.7. Aprile 1992); un lettore trevigiano, pur ammettendo le ragioni del condizionamento dell'italiano da parte dell'inglese nella dominante cultura tecnologica, deplora il necessario e non dignitoso impiego di parole straniere anche in documenti pubblici (CPV, 12.8. Aprile 1996); un infermiere di Còmiso tiene ad esser riconosciuto come «infermiere al 100\%» e non come paramedico, «medico posticcio», glossa il lettore, termine che, scrive ancora, «fa tanto americanismo», ma è palesemente errato e privo di significato (CPV, 41.1. Ottobre 2010).

Pur con il filtro della redazione, siamo certi che termini come liceità, imbastardire, deplorare siano frutto di una veemenza rintracciata nei quesiti; la lettera dell'infermiere è accompagnata, si legge, «da una vivace e documentata requisitoria» (CPV, 41.1. Ottobre 2010).

E, tra chi chiede se si dica lo walkman o il walkman (CPV, 9.5. Ottobre 1994) ${ }^{14}$, chi vuol sapere se e-mail sia femminile o maschile (CPV, 21.7. Ottobre 2000) ${ }^{15}$, e chi segnala che «vadano guadagnando spazio crescente nell'uso contemporaneo, alcuni costrutti che, facoltativi in italiano, sono obbligatori in inglese» (CPV, 2.4. Aprile 1991) ${ }^{16}$. Assai interessanti sono quegli intereventi che pongono il problema della traducibilità dei termini stranieri in italiano.

13. L'argomento e la formula di Castellani ritornano nell'uscita numero 46 del foglio, in cui si riporta un articolo apparso sull'Avvenire del 6 febbraio 2013 firmato da Giacomo Gambassi, dal titolo /l morbus anglicus del signor Rossi, dell'uomo comune prigioniero, ormai, dell'itanglese.

14. Lo stesso quesito numero cinque ospita una richiesta, proveniente da un diverso parlante, sull'uso degli articoli determinativi con parole straniere che iniziano con suono semivocalico o con aspirata.

15. «In inglese il vocabolo è neutro, il solo fatto di attribuire un genere al termine email è già un tratto distintivo di marca della lingua ospitante», risponde Nencioni (CPV, 21.7. Ottobre 2000).

16. Il lettore segnala: stare + gerundio (tipo: stare dormendo); l'aggettivo possessivo in contesti in cui non sarebbe neces- 
Una signora di Parma desidera che le vengano forniti «gli equivalenti italiani delle parole seguenti: brochure, budget, flash, freelance, input, junior (e senior), lay-out, marketing, moonlighter, network, spot, stand» (CPV, 4.7. Aprile 1992); studentessa e impiegata in un'agenzia pubblicitaria vuole sottrarsi «alla moda» dell'imperversare di voci straniere «nel campo della pubblicità» (CPV, 4.7. Aprile 1992). Un lettore torinese parla di «mancanza di dignità popolare», per il superfluo impiego di ticket per biglietto in ambito sanitario, night per locale notturno, trend per tendenza, check per controllo (CPV, 12.8. Aprile 1996); un giornalista sportivo chiede la traducibilità di termini anglosassoni relativi all'automobilismo come team, paddok, pit-lane, motorhome (CPV, 25.12. Ottobre 2002).

\section{I risultati di un questionario}

In corpore vili e senza alcuna pretesa di completezza, per questa seconda e ultima parte, si è preso spunto da alcuni dei forestierismi appena citati per realizzare una lista di termini, che sono stati sottoposti ad un campione di trenta individui (maschi e femmine) divisi in tre fasce d'età A (18-30); B (31-45); C (46-60).

L'esperimento ha voluto verificare se al desiderio dei parlanti di trovare traducenti, corrisponda, sul piano pratico, un'effettiva capacità di individuare termini che assorbano il pieno significato della voce straniera.

Ai nostri intervistati, soggetti ad alto coefficiente di scolarizzazione (23 posseggono una laurea, 7 un diploma di istruzione secondaria superiore), è stato chiesto di fornire una spiegazione del termine straniero e di individuare un corrispettivo italiano, insieme ad alcune domande più generali sulla presenza dei forestierismi nel loro linguaggio quotidiano.

Rispondendo all'apposito quesito, tutti hanno dimostrato di conoscere il significato delle voci presenti nella lista; quando non è stata proposta una sostituzione, si deve dedurre che il termine è ormai acclimato nelle abitudini linguistiche dei nostri parlanti.

I forestierismi sono i seguenti: network, flash, spot, stand, check, trend, budget, ticket, marketing, brochure ${ }^{17}$.

Le tabelle, suddivise per fasce d'età, riportano, per ogni termine, la serie di alternative suggerite e la frequenza nel gruppo di intervistati. Uno schema sinottico sintetizzerà i risultati raggiunti con la voce italiana più frequentemente proposta.

sario (trascorrere le vostre vacanze a...); l'uso del plurale per indicare cose uguali appartenenti a più persone (prendemmo le nostre biciclette) con funzione presumibilmente enfatica; l'impiego dell'aggettivo prima del nome.

17. Della nostra lista di termini, solo ticket figura nel glossario di Giovanardi, Gualdo e Coco (2003) e per l'accezione di «buono, in particolare quello che le aziende rilasciano [...] ai dipendenti per il consumo di pasti a prezzi convenzionati», la sostituzione proposta è buono (Giovanardi, Gualdo e Coco, 2003: 281ss.). 
Tabella 1. Risposte gruppo A

\begin{tabular}{|c|c|c|}
\hline \multicolumn{3}{|c|}{ GRUPPO A (18-30) } \\
\hline Forestierismo & Sostituzioni proposte & Frequenza significati \\
\hline \multirow{4}{*}{ Network } & Rete & 5 \\
\hline & Sistema & 1 \\
\hline & Informazione telematica & 1 \\
\hline & Nessuna sostituzione & 3 \\
\hline \multirow{7}{*}{ Flash } & Abbaglio & 2 \\
\hline & Veloce & 2 \\
\hline & Lampo & 1 \\
\hline & Rapido & 1 \\
\hline & Istante & 1 \\
\hline & Notizia breve & 2 \\
\hline & Nessuna sostituzione & 1 \\
\hline \multirow{3}{*}{ Spot } & Pubblicità & 8 \\
\hline & Messaggio & 1 \\
\hline & Nessuna sostituzione & 1 \\
\hline \multirow{5}{*}{ Stand } & Esposizione & 4 \\
\hline & Attendere & 1 \\
\hline & Spazio operativo & 1 \\
\hline & Banco di vendita & 1 \\
\hline & Nessuna sostituzione & 3 \\
\hline \multirow{2}{*}{ Check } & Controllo & 8 \\
\hline & Nessuna sostituzione & 2 \\
\hline
\end{tabular}


Tabella 1. Risposte gruppo A (continua dalla pagina precedente)

\begin{tabular}{|c|c|c|}
\hline & GRUPPO A (18-30) & \\
\hline \multirow{6}{*}{ Trend } & Oscillazione & 1 \\
\hline & Andamento & 3 \\
\hline & Tendenza & 2 \\
\hline & Variazione & 1 \\
\hline & Moda & 1 \\
\hline & Nessuna sostituzione & 2 \\
\hline \multirow{5}{*}{ Budget } & Valore & 1 \\
\hline & Disponibilità & 2 \\
\hline & Capitale & 2 \\
\hline & Bilancio & 2 \\
\hline & Nessuna sostituzione & 3 \\
\hline \multirow{2}{*}{ Ticket } & Biglietto & 9 \\
\hline & Nessuna sostituzione & 1 \\
\hline \multirow{7}{*}{ Marketing } & Flusso & 1 \\
\hline & Analisi di mercato & 1 \\
\hline & Processo di vendita & 2 \\
\hline & Commercializzazione & 1 \\
\hline & Vendita mirata & 1 \\
\hline & Strategia di vendita & 1 \\
\hline & Nessuna sostituzione & 3 \\
\hline \multirow{6}{*}{ Brochure } & Cartolina & 1 \\
\hline & Opuscolo & 3 \\
\hline & Volantino & 2 \\
\hline & Carta dei servizi & 1 \\
\hline & Depliant & 1 \\
\hline & Nessuna sostituzione & 2 \\
\hline
\end{tabular}


Tabella 2. Risposte gruppo B

\begin{tabular}{|c|c|c|}
\hline \multicolumn{3}{|c|}{ GRUPPO B (31-50) } \\
\hline Forestierismo & Sostituzioni proposte & Frequenza significati \\
\hline \multirow{4}{*}{ Network } & Rete & 3 \\
\hline & Canale televisivo & 2 \\
\hline & Telegiornale & 1 \\
\hline & Nessuna sostituzione & 4 \\
\hline \multirow{4}{*}{ Flash } & Scatto & 1 \\
\hline & Lampo & 4 \\
\hline & Istante & 2 \\
\hline & Nessuna sostituzione & 3 \\
\hline \multirow{3}{*}{ Spot } & Spazio pubblicitario & 7 \\
\hline & Messaggio & 2 \\
\hline & Nessuna sostituzione & 1 \\
\hline \multirow{4}{*}{ Stand } & Spazio dimostrativo & 1 \\
\hline & Spazio espositivo & 3 \\
\hline & Esposizione & 2 \\
\hline & Nessuna sostituzione & 4 \\
\hline \multirow{3}{*}{ Check } & Controllo & 5 \\
\hline & Passo & 1 \\
\hline & Nessuna sostituzione & 4 \\
\hline
\end{tabular}


Tabella 2. Risposte gruppo B (continua dalla pagina precedente)

\begin{tabular}{|c|c|c|}
\hline \multicolumn{3}{|c|}{ GRUPPO B (31-50) } \\
\hline \multirow{5}{*}{ Trend } & Moda & 4 \\
\hline & Moderno & 1 \\
\hline & Tendenza & 3 \\
\hline & Marchio & 1 \\
\hline & Sessuna sostituzione & 1 \\
\hline \multirow{6}{*}{ Budget } & Disponibilità economica & 3 \\
\hline & Fondo monetario & 1 \\
\hline & Limite & 1 \\
\hline & Gruzzolo & 1 \\
\hline & Somma & 1 \\
\hline & Nessuna sostituzione & 3 \\
\hline \multirow{3}{*}{ Ticket } & Titolo & 1 \\
\hline & Biglietto & 6 \\
\hline & Nessuna sostituzione & 3 \\
\hline \multirow{4}{*}{ Marketing } & Area promozionale & 3 \\
\hline & Strategia aziendale & 1 \\
\hline & Pubblicità & 1 \\
\hline & Nessuna sostituzione & 5 \\
\hline \multirow{6}{*}{ Brochure } & Depliant & 3 \\
\hline & Catalogo & 1 \\
\hline & Foglio informativo & 1 \\
\hline & Foglio illustrativo & 2 \\
\hline & Pieghevole & 1 \\
\hline & Nessuna sostituzione & 2 \\
\hline
\end{tabular}


Tabella 3. Risposte gruppo C

\begin{tabular}{|c|c|c|}
\hline \multicolumn{3}{|c|}{ GRUPPO C (51-70) } \\
\hline Forestierismo & Sostituzioni proposte & Frequenza significati \\
\hline \multirow{6}{*}{ Network } & Emittente & 1 \\
\hline & Lavoro informatico & 3 \\
\hline & Rete di parole & 1 \\
\hline & Insieme & 1 \\
\hline & Fonte di informazione & 2 \\
\hline & Nessuna sostituzione & 2 \\
\hline \multirow{4}{*}{ Flash } & Lampo & 5 \\
\hline & Fare qualcosa velocemente & 1 \\
\hline & Istantanea & 1 \\
\hline & Nessuna sostituzione & 3 \\
\hline \multirow{4}{*}{ Spot } & Pubblicità & 3 \\
\hline & Comunicato & 1 \\
\hline & Promozione & 1 \\
\hline & Nessuna sostituzione & 5 \\
\hline \multirow{4}{*}{ Stand } & Padiglione & 3 \\
\hline & Esposizione & 3 \\
\hline & Tenda fieristica & 1 \\
\hline & Nessuna sostituzione & 3 \\
\hline \multirow{3}{*}{ Check } & Controllo & 5 \\
\hline & Indagine & 1 \\
\hline & Nessuna sostituzione & 4 \\
\hline
\end{tabular}


Tabella 3. Risposte gruppo C (continua dalla pagina precedente)

\begin{tabular}{|c|c|c|}
\hline & GRUPPO C (51-70) & \\
\hline \multirow{5}{*}{ Trend } & Tendenza & 3 \\
\hline & Andamento & 1 \\
\hline & Moderno & 1 \\
\hline & Sviluppo di un evento & 1 \\
\hline & Nessuna sostituzione & 4 \\
\hline \multirow{6}{*}{ Budget } & Fondo economico & 2 \\
\hline & Somma stanziata & 1 \\
\hline & Bagaglio & 1 \\
\hline & Disposizione & 1 \\
\hline & Tetto di spesa & 1 \\
\hline & Nessuna sostituzione & 4 \\
\hline \multirow{4}{*}{ Ticket } & Importo & 1 \\
\hline & Biglietto & 6 \\
\hline & Scontrino, segna fila & 1 \\
\hline & Nessuna sostituzione & 2 \\
\hline \multirow{4}{*}{ Marketing } & Tecnica commerciale & 1 \\
\hline & Indagine di mercato & 1 \\
\hline & Scambio & 1 \\
\hline & Nessuna sostituzione & 7 \\
\hline \multirow{5}{*}{ Brochure } & Libretto & 1 \\
\hline & Opuscolo & 3 \\
\hline & Pieghevole & 1 \\
\hline & Depliant & 2 \\
\hline & Nessuna sostituzione & 3 \\
\hline
\end{tabular}


Tabella 4. Schema sinottico delle risposte al questionario

\begin{tabular}{|c|c|c|}
\hline \multicolumn{2}{|c|}{ GRUPPO A (18-30) } & Frequenza \\
\hline Forestierismo & Sostituzioni & 5 \\
\hline Network & Rete & 2 \\
\hline Flash & Veloce & 8 \\
\hline Spot & Pubblicità & 4 \\
\hline Stand & Esposizione & 7 \\
\hline Check & Controllo & 3 \\
\hline Trend & Andamento & 3 \\
\hline Budget & Nessuna sostituzione & 9 \\
\hline Ticket & Biglietto & 3 \\
\hline Marketing & Nessuna sostituzione & 3 \\
\hline Brochure & Opuscolo & \\
\hline
\end{tabular}

\begin{tabular}{|c|c|c|}
\hline \multirow{2}{*}{ Forestierismo } & Sostituzioni & Frequenza \\
\hline Network & Nessuna sostituzione & 4 \\
\hline Flash & Lampo & 4 \\
\hline Spot & Pubblicità & 7 \\
\hline Stand & Nessuna sostituzione & 4 \\
\hline Check & Controllo & 5 \\
\hline Trend & Moda & 4 \\
\hline Budget & Nessuna sostituzione & 3 \\
\hline Ticket & Biglietto & 6 \\
\hline Marketing & Nessuna sostituzione & 5 \\
\hline Brochure & Depliant & 3 \\
\hline
\end{tabular}




\begin{tabular}{|c|c|c|}
\hline \multicolumn{2}{|c|}{ GRUPPO C (46-60) } \\
\hline Forestierismo & Sostituzioni & Frequenza \\
\hline Network & Lavoro Informatico & 5 \\
\hline Flash & Lampo & 5 \\
\hline Spot & Nessuna sostituzione & 3 \\
\hline Stand & Nessuna sostituzione & 4 \\
\hline Check & Nessuna sostituzione & 4 \\
\hline Trend & Nessuna sostituzione & 4 \\
\hline Budget & Nessuna sostituzione & 6 \\
\hline Ticket & Biglietto & 7 \\
\hline Marketing & Nessuna sostituzione & 3 \\
\hline Brochure & Depliant & \\
\hline
\end{tabular}

Qualche riflessione sul questionario.

Il primo gruppo di intervistati non ha mostrato esitazioni a sostituire i termini di riferimento: studenti universitari o giovani laureati, si sono giovati dell'esperienza recente per fornire soluzioni varie, e valide, per termini come check «controllo», stand «esposizione», trend «andamento, tendenza», marketing, per il quale sono state avanzate ben sei diverse proposte («flusso», «analisi di mercato», «processo di vendita', «commercializzazione», «vendita mirata» «strategia di vendita»), network che, senza sorprese, trova il suo corrispettivo in «rete» (per associazione, probabilmente, a social network).

Gli individui appartenenti alla fascia di età intermedia sono quelli che hanno manifestato maggiore difficoltà nella compilazione del questionario: liberi professionisti, insegnanti, operatori sanitari, forse più consapevoli della polisemia delle voci o del diverso contesto d'uso, non hanno nascosto il proprio imbarazzo dinanzi all'incapacità di trovare corrispettivi a parole come budget, check, marcheting, network, stand.

Numerosi sono i termini che il gruppo $\mathrm{C}$ ha ritenuto di non sostituire: probabilmente più radicate nella coscienza del parlante perché accolte da più tempo, le voci straniere coprono a pieno il significato percepito.

Se flash trova un'alternativa, seppur non in maniera univoca, in «lampo», check in 'controllo», per l'anglicismo spot, dagli altri gruppi sostituito con pochi indugi in «pubblicità» (gruppo A) e «spazio pubblicitario» (gruppo B), ben cinque intervistati tra quelli di età più adulta non propongono alcun termine equivalente. In direzione contraria rispetto alle altre fasce, invece, i parlanti del terzo gruppo avanzano per la parola network il maggior numero di proposte, tra le quali figurano: «emittente», «lavoro informatico», «rete di parole», «insieme», «fonte di informazione». 
Se volessimo tracciare delle linee comuni nelle risposte date al questionario, potremmo affermare che il termine meno sostituito è marketing, seguito da budget, stand e check. Di rilievo, invece, la varietà di proposte relative al termine brochure: tra «opuscolo» (che occorre sei volte in totale), «libretto», «pieghevole», sorprende la resa del termine con un altro forestierismo, ovvero depliant, indizio, questo, di come ormai alcuni termini stranieri siano talmente radicati nella nostra lingua e presenti nell'uso quotidiano che possono essere glossati con un'altra voce straniera.

\section{Conclusioni}

Portiamo ancora alcune considerazioni in conclusione generale, al termine di questa rassegna. Il foglio continua ad ospitare richieste che si collocano ai poli dicotomici del giusto/sbagliato ( $\mathrm{Si}$ scrive: volonteroso o volenteroso?», CPV, 34.1. Aprile 2007; «Qual è la resa grafica delle forme flesse di "averci"?», CPV, 36.1. Aprile 2008; «è legittimo "palii" come plurale di "palio"?», CPV, 34.7. Aprile 2007); pressoché immutato è il fastidio per la ridondanza rispetto all’analisi di Serianni 2006 ("“Visione sinottica" è di per sé un pleonasmo?», chiede una lettrice bolognese in CPV, 43.2. Ottobre 2011; ancora, «Perché nella prosa burocratica, scrive una lettrice romana, si parla di "sollecito urgente"? Nel termine "sollecito" non è già implicito il carattere di "urgenza"?», CPV, 38.8. Aprile 2009); ma ciò che di nuovo emerge è un incremento dei quesiti che riguardano il lessico.

A dar prova di ciò, le curiosità che riguardano i linguaggi tecnici-specialistici: il foglio 30 dell'aprile 2005 ospita due richieste di chiarimenti sui termini linguistici apofonia, metafonia e metalessi; una lettrice chiede il significato del termine ipersegno afferente alla critica letteraria (CPV, 32.3. Aprile 2006) e, nello stesso numero, un avvocato segnala il crescente impiego, in ambito giuridico, del verbo comminare nel senso di «infliggere una condanna» (quesito numero 4); per la parola capresteria, usata da Vasari a proposito dei putti del Parmigianino nell'edizione torrentiniana, una lettrice chiede «significato esatto e provenienza» (CPV, 34.12. Aprile 2007).

Lessico, dunque, ma, anche semantica delle parole: il parlante, consapevole che in italiano, come in qualsiasi altra lingua, i sinonimi non esistono, interroga, curioso, gli accademici per cogliere la sfumatura identificativa di un termine rispetto ad un altro e, quel che è più interessante, chiede ragguagli sull'impiego nella pertinente situazione comunicativa, mostra di tenere in considerazione, come già detto, quella variabile diafasica e distratica che diventa centrale nel dibattito sulla lingua proposto dal periodico.

Riguardo la tipologia dei quesiti posti, c'è da chiedersi se, rivolgendosi agli «espertissimi», non sia più o meno inconscio il tentativo di raffinare le richieste, di alzare la soglia di sorveglianza rispetto all'errore, alla difformità, alla deviazione dalla norma; potrebbe essere legittimo pensare che, dietro certo purismo imperante, dietro la difesa strenua della lingua nazionale rispetto alla penetrazione dei forestierismi, (che per gli utenti della Crusca per voi sembra poggiare sull'assunto: «Se esiste una voce italiana, perché usare quella straniera?»), ci sia una sorta di tentativo di assecondare delle ideologie, 
proprie di un'istituzione autorevole come quella dell'Accademia della Crusca, che si immaginano, erroneamente, essere conservative e prescrittive.

Il conservatorismo individuato nei lettori del periodico, non sembra, invece, animare gli intervistati al nostro questionario: probabilmente liberi da ogni condizionamento, forti dell'anonimato dell'intervista e consapevoli che non sarebbero stati giudicati da un gruppo di esperti, non hanno nascosto la difficoltà ad individuare un corrispettivo italiano in grado di inglobare a pieno le caratteristiche semantiche, sociali, culturali che riveste la voce straniera ormai comune.

In realtà, anche l'opinione degli studiosi riguardo il grado di accettabilità dei forestierismi in italiano è andata via via modificandosi : dal già citato morbus anglicus di Castellani, che, nelle pagine della stessa Crusca per voi, nel 1992, asseriva: «Secondo me nessun problema è più grave, per l'italiano di oggi, di quello degli anglicismi», alla cautela di Serianni per il quale «molte volte il termine straniero risponde a una semplice ostentazione snobistica e rivela, più che le aperture internazionali, la pigrizia, il provincialismo e magari la superficiale cultura dello scrivente» (CPV, 1.7-9. Ottobre 1990), fino ad una certa apertura in Nencioni che avverte:

Se ne avessi l'autorità, vorrei chiedere agli autori di dizionari e grammatiche di curare in modo particolare il tema dei forestierismi, orientando i lettori sul loro uso, se necessario o opportuno, e sulla loro intelligente associazione alla nostra lingua, che di essi si è facilmente arricchita fino dall'antichità classica (come l'inglese, lingua germanica, si è arricchito di elementi francesi e latini). (CPV, 21.7 Ottobre 2000)

La nostra indagine conferma, dunque, la «salutare sensibilità - o ipersensibilità» (Serianni, 2006: 54) dei parlanti nei confronti della lingua nazionale, ma, seppur sia ancora viva la percezione monolitica della norma tradizionale, nella quale gli utenti «identificano la tenuta stessa della lingua» (Antonelli, 2007: 43), sembra avanzare, lentamente, ma significativamente (e anche il dato sintetico del questionario con un numero cospicuo di termini non sostituiti lo dimostra), la consapevolezza del mutare della lingua, della spinta che le viene ormai da ogni direzione e che la conferma, per dirla ancora con Nencioni, «una realtà naturale, della naturalità propria della cultura» (Nencioni, 1992, CPV). 


\section{Bibliografia}

Accademia della Crusca (ed.) (2005), La Crusca risponde, Firenze, Le Lettere.

Antonelli, Giuseppe (2007), L'italiano nella società della comunicazione, Bologna, Il Mulino.

Carrera Díaz, Manuel (2000), «Forestierismi. Norma italiana e norme europee», in Serge Vanvolsem et al. (ed.), L'italiano oltre frontiera. V Convegno internazionale, Leuven, 22-25 aprile 1998, Leuven/ Firenze, University Press/Cesati, p. 19-29.

Coletti, Vittorio (1989), Italiano d'autore. Saggi di lingua e letteratura del Novecento, Genova, Marietti.

Coseriu, Eugenio (1969), «Sistema, norma e "parola"», in Studi linguistici in onore di Vittore Pisani, Brescia, Paideia, vol. 1, p. 235-254.

Coseriu, Eugenio (1971), Teoria del linguaggio e linguistica generale, Bari, Laterza.

CVP: La Crusca pervoi, Firenze, Accademia della Crusca, 1990-.

Giovanardi, Claudio, Riccardo Gualdo e Alessandra Coco (2003), Inglese-Italiano 1 a 1. Tradurre o non tradurre le parole inglesi, Lecce, Manni.

Marazzini, Claudio (2006), «Sulla norma dell'italiano moderno. Con una riflessione sull'origine e sulla legittimità delle "regole" secondo gli antichi grammatici», Lid'O. Lingua italiana d'Oggi, n 3, p. 85-101.

Migliorini, Bruno (1975), Parole d'autore. Onomaturgo, Firenze, Sansoni.

Nencioni, Giovanni (1989), Saggi di lingua e antica e moderna, Torino, Rosenberg e Sellier.

Palermo, Massimo (1995), «I manuali redazionali e la norma nell'italiano scritto contemporaneo», Studi linguistici italiani, n² 21, p. 88-115.

Pontiggia, Giuseppe (2009), Prima persona, Milano, Mondadori.

Renzi, Lorenzo (2012), Come cambia la lingua. L'italiano in movimento, Bologna, Il Mulino.

Rossi, Fabio e Fabio Ruggiano (2013), Scrivere in italiano. Dalla pratica alla teoria, Roma, Carocci.

Serianni, Luca (2004), «ll sentimento della norma linguistica nell'Italia di oggi», Studi linguistici italiani, n³0, p. 85-103.

Serianni, Luca (2006), Prima lezione di grammatica, Bari, Laterza.

Serianni, Luca (2007), «La norma sommersa», Lingua e stile, n 42, p. 283-298.

Serianni, Luca (2008), «La norma e la scuola. Primi materiali», in Anna Ciliberti (ed.), Un mondo di italiano, Perugia, Guerra, p. 61-80. 
Serianni, Luca (2014), «Giusto o sbagliato. Dove comincia il territorio dell'errore?», in Sergio Lubello (ed.), Lezioni d'italiano. Riflessioni sulla lingua del nuovo millennio, Bologna, Il Mulino, p. 235-246.

Sgroi, Salvatore Claudio (2010), Per una grammatica «laica». Esercizi di analisi linguistica dalla parte del parlante, Torino, Utet. 